\title{
Asymptotic non-degeneracy of the solution to the Liouville-Gel'fand problem in two dimensions
}

Tomohiko Sato and Takashi Suzuki

Abstract. In this paper we study the asymptotic non-degeneracy of the solution to the LiouvilleGel'fand problem

$$
-\Delta v=\lambda V(x) e^{v} \text { in } \Omega, \quad v=0 \text { on } \partial \Omega,
$$

where $\Omega \subset \mathbb{R}^{2}$ is a smooth bounded domain, $V(x)$ is a positive-valued $C^{1}(\bar{\Omega})$ function, and $\lambda>0$ is a constant.

Mathematics Subject Classification (2000). 35J60.

Keywords. Liouville equation, blow-up analysis, Green's function.

\section{Introduction}

The purpose of the present paper is to study the asymptotic non-degeneracy of the solution to the Liouville-Gel'fand problem

$$
-\Delta v=\lambda V(x) e^{v} \text { in } \Omega, \quad v=0 \text { on } \partial \Omega,
$$

where $\Omega \subset \mathbb{R}^{2}$ is a bounded domain with smooth boundary $\partial \Omega, V=V(x)>0$ is a $C^{1}$ function defined on $\bar{\Omega}$, and $\lambda>0$ is a constant. We shall extend a result of Gladiali-Grossi [5], which is valid for the homogeneous case of $V(x) \equiv 1$,

$$
-\Delta v=\lambda e^{v} \text { in } \Omega, \quad v=0 \text { on } \partial \Omega
$$

based on the following fact [8].

Theorem 1.1. If $\left(\lambda_{k}, v_{k}\right)(k=1,2, \ldots)$ is a solution sequence for (2) satisfying $\lambda_{k} \rightarrow 0$, then we have a subsequence (denoted by the same symbol) such that $\Sigma_{k}=$ $\int_{\Omega} \lambda_{k} e^{v_{k}} \rightarrow 8 \pi m$ for some $m=0,1,2, \ldots,+\infty$. According to this value of $m$, we have the following.

(1) If $m=0$, then it holds that $\left\|v_{k}\right\|_{\infty} \rightarrow 0$. 
(2) If $0<m<+\infty$, then the blowup set of $v_{k}(k=1,2, \ldots)$, defined by

$$
\delta=\left\{x_{0} \in \bar{\Omega} \mid \text { there exists } x_{k} \rightarrow x_{0} \text { such that } v_{k}\left(x_{k}\right) \rightarrow+\infty\right\},
$$

is composed of m-interior points, and $v_{k} \rightarrow 8 \pi \sum_{x_{0} \in \delta} G\left(\cdot, x_{0}\right)$ locally uniformly in $\bar{\Omega} \backslash \delta$, where $G=G(x, y)$ denotes the Green's function of $-\Delta$ in $\Omega$ with $\left.\cdot\right|_{\partial \Omega}=0$. We have $-\Delta v_{k}(x) d x \rightarrow \sum_{x_{0} \in \delta} 8 \pi \delta_{x_{0}}(d x)$ in the sense of measure on $\bar{\Omega}$. Furthermore, it holds that

$$
\frac{1}{2} \nabla R\left(x_{0}\right)+\sum_{x_{0}^{\prime} \in S \backslash\left\{x_{0}\right\}} \nabla_{x} G\left(x_{0}, x_{0}^{\prime}\right)=0
$$

for each $x_{0} \in \delta$, where $R(x)=\left[G(x, y)+\frac{1}{2 \pi} \log |x-y|\right]_{y=x}$ is the Robin function.

(3) If $m=+\infty$, then $v_{k} \rightarrow+\infty$ locally uniformly in $\Omega$.

Gladiali and Grossi [5] are concerned with the case $m=1$, and study the nondegeneracy of $\left(\lambda_{k}, v_{k}\right)$ for large $k$. From the above theorem, we have $s=\left\{x_{0}\right\}$ if $m=1$ and this $x_{0} \in \Omega$ is a critical point of the Robin function. What they obtained is the following theorem, motivated by the study of the detailed bifurcation diagram for (2).

Theorem 1.2. If $m=1$ holds in the previous theorem and $x_{0} \in s$ is a non-degenerate critical point of $R(x)$, then the solution $\left(\lambda_{k}, v_{k}\right)$ is non-degenerate for large $k$, that is, the linearized operator $-\Delta-\lambda_{k} e^{v_{k}}$ in $\Omega$ with $\left.\cdot\right|_{\partial \Omega}=0$ is invertible.

Theorem 1.1, on the other hand, has an extension to (1). Although the results of Ma-Wei [7] are presented in the mean field formulation,

$$
-\Delta v=\frac{\lambda V(x) e^{v}}{\int_{\Omega} V(x) e^{v}} \quad \text { in } \Omega, \quad v=0 \text { on } \partial \Omega,
$$

it is easy to translate them into the following theorem on (1). (See also [9].)

Theorem 1.3. All the results stated in Theorem 1.1 continue to hold for (1), provided that $\Sigma_{k}$ and (3) are replaced by $\Sigma_{k}=\int_{\Omega} \lambda_{k} V(x) e^{v_{k}}$ and

$$
\frac{1}{2} \nabla R\left(x_{0}\right)+\sum_{x_{0}^{\prime} \in S \backslash\left\{x_{0}\right\}} \nabla_{x} G\left(x_{0}, x_{0}^{\prime}\right)+\frac{1}{8 \pi} \nabla \log V\left(x_{0}\right)=0,
$$

respectively. 
In the case of $m=1$ again, equation (4) means that $x_{0} \in \Omega$ is a critical point of $R(x)+\frac{1}{4 \pi} \log V(x)$. From this point of view, it is natural to extend Theorem 1.2 as follows.

Theorem 1.4. In Theorem 1.3, if $m=1, V(x)$ is $C^{2}$ near $x_{0} \in 8$, and $x_{0}$ is a non-degenerate critical point of $R(x)+\frac{1}{4 \pi} \log V(x)$, then the solution $\left(\lambda_{k}, v_{k}\right)$ is non-degenerate for large $k$, that is, the linearized operator $-\Delta-\lambda_{k} V(x) e^{v_{k}}$ in $\Omega$ with $\left.\cdot\right|_{\partial \Omega}=0$ is invertible.

To prove the above theorem, we follow the argument of [5], namely, the existence of $w_{k}=w_{k}(x)(k=1,2, \ldots)$ satisfying

$$
\begin{aligned}
-\Delta w_{k} & =\lambda_{k} V(x) e^{v_{k}} w_{k} \text { in } \Omega, \quad w_{k}=0 \text { on } \partial \Omega, \\
\left\|w_{k}\right\|_{\infty} & =1,
\end{aligned}
$$

implies a contradiction. The next section is devoted to examine the validity of the blowup analysis [5] to (1), originally developed for (2). In the latter case, $w_{k}^{\prime}=\frac{\partial v_{k}}{\partial x_{i}}$ $(i=1,2)$ solves the linearized equation

$$
-\Delta w_{k}^{\prime}=\lambda_{k} e^{v_{k}} w_{k}^{\prime} \quad \text { in } \Omega
$$

(except for the boundary condition). This structure is useful to prove Theorem 1.2, but obviously does not hold in (1). In the final section, we complete the proof of Theorem 1.4, providing new arguments to compensate this obstruction.

\section{Preliminaries}

In this section, we confirm that several assertions for (2) presented in [5] are still valid for (1). Henceforth, $\left(\lambda_{k}, v_{k}\right)(k=1,2, \ldots)$ is a solution sequence for (1) satisfying

$$
\Sigma_{k}=\int_{\Omega} \lambda_{k} V(x) e^{v_{k}} \rightarrow 8 \pi, \quad \lambda_{k} \rightarrow 0,
$$

and $x_{k} \in \Omega$ denotes a maximum point of $v_{k}$ :

$$
v_{k}\left(x_{k}\right)=\left\|v_{k}\right\|_{\infty} .
$$

Then we have $x_{k} \rightarrow x_{0}$ with $\delta=\left\{x_{0}\right\}$, and this blowup point $x_{0} \in \Omega$ is a critical point of $R(x)+\frac{1}{4 \pi} \log V(x)$.

The first lemma corresponds to Theorem 6 of [5].

Lemma 2.1. There is a constant $C_{1}>0$ such that

$$
\left|v_{k}(x)-\log \frac{e^{v_{k}\left(x_{k}\right)}}{\left\{1+\frac{1}{8} \lambda_{k} V\left(x_{k}\right) e^{v_{k}\left(x_{k}\right)}\left|x-x_{k}\right|^{2}\right\}^{2}}\right| \leq C_{1}
$$

for any $x \in \bar{\Omega}$ and $k=1,2, \ldots$ 
Proof. Putting $u_{k}=v_{k}+\log \lambda_{k}$, we obtain

$$
\begin{aligned}
-\Delta u_{k} & =V(x) e^{u_{k}} \text { in } \Omega, \quad u_{k}=\log \lambda_{k} \text { on } \partial \Omega, \\
\int_{\Omega} e^{u_{k}} & =O(1) .
\end{aligned}
$$

Passing to a subsequence, we shall show that $u_{k}\left(x_{k}\right) \rightarrow+\infty$ holds. Then, Theorem 0.3 of Y. Y. Li [6] guarantees the existence of $C_{1}>0$ such that

$$
\left|u_{k}(x)-\log \frac{e^{u_{k}\left(x_{k}\right)}}{\left\{1+\frac{1}{8} V\left(x_{k}\right) e^{u_{k}\left(x_{k}\right)}\left|x-x_{k}\right|^{2}\right\}^{2}}\right| \leq C_{1}
$$

for any $x \in \bar{\Omega}$ and $k=1,2, \ldots$, or, equivalently, (7).

In fact, if $u_{k}\left(x_{k}\right) \rightarrow+\infty$ does not occur, then we may assume either $u_{k}\left(x_{k}\right) \rightarrow$ $-\infty$ or $u_{k}\left(x_{k}\right) \rightarrow c \in \mathbb{R}$. In the first alternative, we have

$$
\int_{\Omega} \lambda_{k} e^{v_{k}} \rightarrow 0
$$

which is impossible by (6), because there are $a, b>0$ such that

$$
a \leq V(x) \leq b \quad(x \in \bar{\Omega}) .
$$

In the second alternative, on the other hand, the sequence $\left\{u_{k}\right\}$ is locally uniformly bounded in $\Omega$ by Brezis-Merle [1], while Theorem 1.3 guarantees $u_{k}=v_{k}+\log \lambda_{k} \rightarrow$ $-\infty$ locally uniformly in $\bar{\Omega} \backslash\left\{x_{0}\right\}$. Again, we have a contradiction, and the proof is complete.

Now we define $\delta_{k}>0$ by

$$
\delta_{k}^{2} \lambda_{k} e^{v_{k}\left(x_{k}\right)}=1 .
$$

The next lemma corresponds to Lemma 5 of [5].

Lemma 2.2. It holds that $\delta_{k} \rightarrow 0$.

Proof. Inequality (7) reads

$$
\left|v_{k}(x)-v_{k}\left(x_{k}\right)+\log \left\{1+\frac{V\left(x_{k}\right)}{8 \delta_{k}^{2}}\left|x-x_{k}\right|^{2}\right\}^{2}\right| \leq C_{1}
$$

for $x \in \bar{\Omega}$ and $k=1,2, \ldots$, and we have $v_{k} \rightarrow 8 \pi G\left(\cdot, x_{0}\right)$ locally uniformly in $\bar{\Omega} \backslash\left\{x_{0}\right\}, V\left(x_{k}\right) \rightarrow V\left(x_{0}\right)$, and $v_{k}\left(x_{k}\right) \rightarrow+\infty$. These imply $\delta_{k} \rightarrow 0$, because otherwise we have a contradiction. 
We assume the existence of $w_{k}=w_{k}(x)$ satisfying (5) and derive a contradiction. For this purpose, we put

$$
\begin{aligned}
\tilde{v}_{k}(x) & =v_{k}\left(x_{k}+\delta_{k} x\right)-v_{k}\left(x_{k}\right), \\
\tilde{w}_{k}(x) & =w_{k}\left(x_{k}+\delta_{k} x\right), \\
\tilde{V}_{k}(x) & =V\left(x_{k}+\delta_{k} x\right),
\end{aligned}
$$

where $x \in \tilde{\Omega}_{k}$ for $\tilde{\Omega}_{k}=\left\{x \in \mathbb{R}^{2} \mid x_{k}+\delta_{k} x \in \Omega\right\}$. We have

$$
\begin{aligned}
-\Delta \tilde{v}_{k} & =\tilde{V}_{k} e^{\tilde{v}_{k}}, \quad \tilde{v}_{k} \leq 0=\tilde{v}_{k}(0) \text { in } \tilde{\Omega}_{k}, \\
\int_{\tilde{\Omega}_{k}} e^{\tilde{v}_{k}} & =\int_{\Omega} \lambda_{k} e^{v_{k}} \leq C_{2}
\end{aligned}
$$

with a constant $C_{2}>0$ independent of $k$, and

$$
\begin{aligned}
-\Delta \tilde{w}_{k} & =\tilde{V}_{k} e^{\tilde{v}_{k}} \tilde{w}_{k} \text { in } \tilde{\Omega}_{k}, \quad \tilde{w}_{k}=0 \text { on } \partial \tilde{\Omega}_{k}, \\
\left\|\tilde{w}_{k}\right\|_{\infty} & =1 .
\end{aligned}
$$

Concerning $\tilde{v}_{k}$, we can apply [1]. Thus, passing to a subsequence, we obtain $\tilde{v}_{k} \rightarrow \tilde{v}_{0}$ in $C_{\text {loc }}^{2, \alpha}\left(\mathbb{R}^{2}\right)$ for $0<\alpha<1$, with $\tilde{v}_{0}=\tilde{v}_{0}(x)$ satisfying

$$
-\Delta \tilde{v}_{0}=V\left(x_{0}\right) e^{\tilde{v}_{0}}, \quad \tilde{v}_{0} \leq 0=\tilde{v}_{0}(0) \text { in } \mathbb{R}^{2}, \quad \int_{\mathbb{R}^{2}} e^{\tilde{v}_{0}}<+\infty,
$$

and therefore

$$
\tilde{v}_{0}(x)=\log \frac{1}{\left\{1+\frac{1}{8} V\left(x_{0}\right)|x|^{2}\right\}^{2}}
$$

by [4]. This implies $\tilde{w}_{k} \rightarrow \tilde{w}_{0}$ in $C_{\text {loc }}^{2, \alpha}\left(\mathbb{R}^{2}\right)$ for a subsequence, with $\tilde{w}_{0}=\tilde{w}_{0}(x)$ satisfying

$$
\begin{aligned}
& -\Delta \tilde{w}_{0}=V\left(x_{0}\right) e^{\tilde{v}_{0}} \tilde{w}_{0}=\frac{V\left(x_{0}\right)}{\left\{1+\frac{1}{8} V\left(x_{0}\right)|x|^{2}\right\}^{2}} \tilde{w}_{0} \text { in } \mathbb{R}^{2}, \\
& \left\|\tilde{w}_{0}\right\|_{\infty} \leq 1 .
\end{aligned}
$$

We shall show $\tilde{w}_{0}=0$ in $\mathbb{R}^{2}$. In fact, if this is the case, then it holds that $\left|y_{k}\right| \rightarrow+\infty$, where $y_{k} \in \tilde{\Omega}_{k}$ denotes a maximum point of $\tilde{w}_{k}=\tilde{w}_{k}(x) ; \tilde{w}_{k}\left(y_{k}\right)=$ $\left\|\tilde{w}_{k}\right\|_{\infty}=1$. We make the Kelvin transformation

$$
\hat{v}_{k}(x)=\tilde{v}_{k}\left(\frac{x}{|x|^{2}}\right), \quad \hat{w}_{k}(x)=\tilde{w}_{k}\left(\frac{x}{|x|^{2}}\right),
$$


and obtain

$$
\begin{aligned}
\left\|\hat{w}_{k}\right\|_{\infty} & =\hat{w}_{k}\left(\frac{y_{k}}{\left|y_{k}\right|^{2}}\right)=1, \\
-\Delta \hat{w}_{k} & =\frac{1}{|x|^{4}} \tilde{V}_{k}\left(\frac{x}{|x|^{2}}\right) e^{\hat{v}_{k}} \hat{w}_{k} \quad \text { in } B_{1}(0) \backslash\{0\}
\end{aligned}
$$

for large $k$. On the other hand, inequality (7) reads

$$
\left|\tilde{v}_{k}(x)+\log \left\{1+\frac{1}{8} V\left(x_{k}\right)|x|^{2}\right\}^{2}\right| \leq C_{1}
$$

for $x \in \tilde{\Omega}_{k}$ and $k=1,2, \ldots$, and we have $e^{\tilde{v}_{k}(x)}=O\left(\frac{1}{|x|^{4}}\right)$ uniformly in $k$. This means $\frac{1}{|x|^{4}} e^{\hat{v}_{k}(x)}=O(1)$ uniformly in $k$, and therefore $x=0$ is a removable singularity of $\hat{w}_{k}$,

$$
-\Delta \hat{w}_{k}=a_{k}(x) \hat{w}_{k} \quad \text { in } B_{1}(0)
$$

with $a_{k}=a_{k}(x)$ satisfying $\left\|a_{k}\right\|_{L^{\infty}\left(B_{1}(0)\right)}=O(1)$. Then, the local elliptic estimate guarantees $1=\left\|\hat{w}_{k}\right\|_{L^{\infty}\left(B_{1 / 2}(0)\right)} \leq C\left\|\hat{w}_{k}\right\|_{L^{2}\left(B_{1}(0)\right)}$, where the right-hand side converges to 0 by the dominated convergence theorem. This is a contradiction and we obtain the proof of Theorem 1.4.

To prove $\tilde{w}_{0}=0$ in $\mathbb{R}^{2}$, we put $c=V\left(x_{0}\right)>0$ and $v(x)=\tilde{w}_{0}(x / \sqrt{c})$ in (9). Then, this $v=v(x) \in L^{\infty}\left(\mathbb{R}^{2}\right)$ satisfies

$$
-\Delta v=\frac{v}{\left\{1+\frac{1}{8}|x|^{2}\right\}^{2}} \quad \text { in } \mathbb{R}^{2}
$$

and hence it holds that

$$
v(x)=\sum_{i=1}^{2} \frac{a_{i} x_{i}}{8+|x|^{2}}+b \cdot \frac{8-|x|^{2}}{8+|x|^{2}}
$$

by [2], where $a_{i}, b \in \mathbb{R}$. Thus, we only have to derive $a_{i}=b=0$ in

$$
\tilde{w}_{0}(x)=\sum_{i=1}^{2} \frac{a_{i} x_{i}}{\frac{8}{c}+|x|^{2}}+b \cdot \frac{\frac{8}{c}-|x|^{2}}{\frac{8}{c}+|x|^{2}} .
$$

We note that $a_{i} / \sqrt{c}\left(a_{i}\right.$ in the formula for $\left.v(x)\right)$ is newly denoted by $a_{i}$.

To show $a_{i}=0$, we use the following lemma, proven similarly to (3.13) in [5].

Lemma 2.3. In case $\left(a_{1}, a_{2}\right) \neq(0,0)$, it holds that

$$
\delta_{k}^{-1} w_{k}(x)=2 \pi \sum_{j=1}^{2} a_{j} \frac{\partial G}{\partial y_{j}}\left(x, x_{0}\right)+o(1)
$$

locally uniformly in $x \in \bar{\Omega} \backslash\left\{x_{0}\right\}$. 
Proof. In fact, we have

$$
\begin{aligned}
w_{k}(x) & =\int_{\Omega} G(x, y) \lambda_{k} V(y) e^{v_{k}(y)} w_{k}(y) d y \\
& =\int_{\tilde{\Omega}_{k}} G\left(x, x_{k}+\delta_{k} y^{\prime}\right) \tilde{V}_{k}\left(y^{\prime}\right) e^{\tilde{v}_{k}\left(y^{\prime}\right)} \tilde{w}_{k}\left(y^{\prime}\right) d y^{\prime}=\mathrm{I}_{1, k}(x)+\mathrm{I}_{2, k}(x),
\end{aligned}
$$

where

$$
\begin{aligned}
& \mathrm{I}_{1, k}(x)=\int_{\tilde{\Omega}_{k}} G\left(x, x_{k}+\delta_{k} y^{\prime}\right) \cdot f_{k}\left(y^{\prime}\right) d y^{\prime} \\
& \mathrm{I}_{2, k}(x)=\int_{\tilde{\Omega}_{k}} G\left(x, x_{k}+\delta_{k} y^{\prime}\right) \cdot \frac{64 b}{c} \cdot \frac{\frac{8}{c}-\left|y^{\prime}\right|^{2}}{\left(\frac{8}{c}+\left|y^{\prime}\right|^{2}\right)^{3}} d y^{\prime}
\end{aligned}
$$

with

$$
f_{k}(y)=\tilde{V}_{k}(y) e^{\tilde{v}_{k}(y)} \tilde{w}_{k}(y)-\frac{64 b}{c} \cdot \frac{\frac{8}{c}-|y|^{2}}{\left(\frac{8}{c}+|y|^{2}\right)^{3}} .
$$

We have

$$
\tilde{V}_{k}(y) e^{\tilde{v}_{k}(y)} \tilde{w}_{k}(y) \rightarrow c \cdot \frac{1}{\left(1+\frac{c}{8}|y|^{2}\right)^{2}} \cdot\left(\sum_{i=1}^{2} \frac{a_{i} y_{i}}{\frac{8}{c}+|y|^{2}}+b \cdot \frac{\frac{8}{c}-|y|^{2}}{\frac{8}{c}+|y|^{2}}\right),
$$

or equivalently,

$$
f_{k}(y) \rightarrow f_{0}(y)=\frac{64}{c} \sum_{i=1}^{2} \frac{a_{i} y_{i}}{\left(\frac{8}{c}+|y|^{2}\right)^{3}},
$$

locally uniformly in $y \in \mathbb{R}^{2}$.

We have, on the other hand, $f_{k}(y)=O\left(\frac{1}{|y|^{4}}\right)$ uniformly in $k=1,2, \ldots$ by (10), and therefore $g_{k}(y) \rightarrow g_{0}(y)$ locally uniformly in $y \in \mathbb{R}^{2}$ by the dominated convergence theorem, where

$$
g_{k}\left(y_{1}, y_{2}\right)=-\int_{\frac{a_{1} y_{1}+a_{2} y_{2}}{a_{1}^{2}+a_{2}^{2}}}^{+\infty} f_{k}\left(a_{1} t+\frac{a_{2}^{2} y_{1}-a_{1} a_{2} y_{2}}{a_{1}^{2}+a_{2}^{2}}, a_{2} t-\frac{a_{1} a_{2} y_{1}-a_{1}^{2} y_{2}}{a_{1}^{2}+a_{2}^{2}}\right) d t
$$

for $k=0,1,2, \ldots$ This $g_{k}$, introduced in Lemma 6 of [5], satisfies

$$
a_{1} \frac{\partial g_{k}}{\partial y_{1}}+a_{2} \frac{\partial g_{k}}{\partial y_{2}}=f_{k}
$$


and therefore it holds that

$$
\begin{aligned}
\mathrm{I}_{1, k}(x) & =\int_{\tilde{\Omega}_{k}} G\left(x, x_{k}+\delta_{k} y^{\prime}\right) f_{k}\left(y^{\prime}\right) d y^{\prime} \\
& =\int_{\tilde{\Omega}_{k}} G\left(x, x_{k}+\delta_{k} y^{\prime}\right) \cdot \sum_{j=1}^{2} a_{j} \frac{\partial g_{k}}{\partial y_{j}^{\prime}}\left(y^{\prime}\right) d y^{\prime} \\
& =-\delta_{k} \sum_{j=1}^{2} a_{j} \int_{\tilde{\Omega}_{k}} \frac{\partial G}{\partial y_{j}}\left(x, x_{k}+\delta_{k} y^{\prime}\right) \cdot g_{k}\left(y^{\prime}\right) d y^{\prime} \\
& =\delta_{k}\left\{\sum_{j=1}^{2} a_{j} \frac{\partial G}{\partial y_{j}}\left(x, x_{0}\right) \int_{\mathbb{R}^{2}} \frac{16}{c} \cdot \frac{1}{\left(\frac{8}{c}+\left|y^{\prime}\right|^{2}\right)^{2}} d y^{\prime}+o(1)\right\} \\
& =\delta_{k}\left\{2 \pi \sum_{j=1}^{2} a_{j} \frac{\partial G}{\partial y_{j}}\left(x, x_{0}\right)+o(1)\right\}
\end{aligned}
$$

locally uniformly in $x \in \bar{\Omega} \backslash\left\{x_{0}\right\}$ by the dominated convergence theorem.

To study $\mathrm{I}_{2, k}(x)$, we note that $u(y)=\log \frac{64}{c} \cdot \frac{1}{\left(\frac{8}{c}+|y|^{2}\right)^{2}}$ satisfies

$$
\frac{\partial}{\partial y_{1}}\left(y_{1} e^{u}\right)+\frac{\partial}{\partial y_{2}}\left(y_{2} e^{u}\right)=\frac{128}{c} \cdot \frac{\frac{8}{c}-|y|^{2}}{\left(\frac{8}{c}+|y|^{2}\right)^{3}},
$$

and in this case we obtain

$$
\begin{aligned}
\mathrm{I}_{2, k}(x) & =\left.\frac{b}{2} \int_{\tilde{\Omega}_{k}} G\left(x, x_{k}+\delta_{k} y^{\prime}\right) \cdot \sum_{j=1}^{2} \frac{\partial}{\partial y_{j}}\left(y_{j} e^{u(y)}\right)\right|_{y=y^{\prime}} d y^{\prime} \\
& =-\delta_{k} \frac{b}{2} \sum_{j=1}^{2} \int_{\tilde{\Omega}_{k}} \frac{\partial G}{\partial y_{j}}\left(x, x_{k}+\delta_{k} y^{\prime}\right) \cdot y_{j}^{\prime} e^{u\left(y^{\prime}\right)} d y^{\prime} \\
& =-\delta_{k} \frac{b}{2}\left\{\sum_{j=1}^{2} \frac{\partial G}{\partial y_{j}}\left(x, x_{0}\right) \cdot \int_{\mathbb{R}^{2}} y_{j}^{\prime} e^{u\left(y^{\prime}\right)} d y^{\prime}+o(1)\right\}=o\left(\delta_{k}\right)
\end{aligned}
$$

locally uniformly in $x \in \bar{\Omega} \backslash\left\{x_{0}\right\}$, again by the dominated convergence theorem. Thus, the proof of (11) is complete.

\section{Proof of Theorem 1.4}

We prove the following lemma, using new arguments. 
Vol. 82 (2007)

Lemma 3.1. If $V(x)$ is $C^{2}$ near $x=x_{0} \in \Omega$ and $x_{0}$ is a non-degenerate critical point of $R(x)+\frac{1}{4 \pi} \log V(x)$, then it holds that $a_{1}=a_{2}=0$.

Proof. We suppose the contrary, and then obtain (11) locally uniformly in $x \in \bar{\Omega} \backslash\left\{x_{0}\right\}$. We note

$$
-\Delta \frac{\partial v_{k}}{\partial x_{i}}=\lambda_{k} V e^{v_{k}} \frac{\partial v_{k}}{\partial x_{i}}+\lambda_{k} V e^{v_{k}} \frac{\partial \log V}{\partial x_{i}} \text { in } \Omega
$$

and define $h_{i, k}=h_{i, k}(x)$ by

$$
-\Delta h_{i, k}=\frac{\partial \log V}{\partial x_{i}} \cdot \lambda_{k} V e^{v_{k}} \text { in } \Omega, \quad h_{i, k}=0 \text { on } \partial \Omega,
$$

where $i=1,2$. Then it follows that

$$
w_{k} \Delta\left(\frac{\partial v_{k}}{\partial x_{i}}-h_{i, k}\right)-\Delta w_{k} \cdot \frac{\partial v_{k}}{\partial x_{i}}=0 \quad \text { in } \Omega
$$

by (5), and therefore we have

$$
\int_{\partial \Omega}\left\{w_{k} \frac{\partial}{\partial v}\left(\frac{\partial v_{k}}{\partial x_{i}}-h_{i, k}\right)-\frac{\partial w_{k}}{\partial v} \cdot\left(\frac{\partial v_{k}}{\partial x_{i}}-h_{i, k}\right)\right\}=\int_{\Omega} h_{i, k} \Delta w_{k} .
$$

Here and henceforth, $v$ denotes the outer unit normal vector on $\partial \Omega$. Since $w_{k}=$ $h_{i, k}=0$ on $\partial \Omega$, the above equation is reduced to

$$
\begin{aligned}
\delta_{k}^{-1} \int_{\partial \Omega} \frac{\partial v_{k}}{\partial x_{i}} \frac{\partial w_{k}}{\partial v} & =-\delta_{k}^{-1} \int_{\Omega} h_{i, k} \Delta w_{k}=-\delta_{k}^{-1} \int_{\Omega} \Delta h_{i, k} \cdot w_{k} \\
& =\delta_{k}^{-1} \int_{\Omega} \frac{\partial \log V}{\partial x_{i}} \cdot \lambda_{k} V e^{v_{k}} \cdot w_{k} .
\end{aligned}
$$

We have

$$
\begin{array}{rlrl}
v_{k} & \rightarrow 8 \pi G\left(\cdot, x_{0}\right) & & \text { in } C_{\mathrm{loc}}^{2, \alpha}\left(\bar{\Omega} \backslash\left\{x_{0}\right\}\right), \\
\delta_{k}^{-1} w_{k} & \rightarrow 2 \pi \sum_{j=1}^{2} a_{j} \frac{\partial G}{\partial y_{j}}\left(\cdot, x_{0}\right) & \text { in } C_{\mathrm{loc}}^{2, \alpha}\left(\bar{\Omega} \backslash\left\{x_{0}\right\}\right)
\end{array}
$$

by Theorem 1.3 and the elliptic estimate, and therefore the left-hand side of (12) converges to

$$
16 \pi^{2} \sum_{j=1}^{2} a_{j} \int_{\partial \Omega} \frac{\partial G}{\partial x_{i}}\left(x, x_{0}\right) \frac{\partial^{2} G}{\partial y_{j} \partial v_{x}}\left(x, x_{0}\right) .
$$

Now we apply Lemma 7 of [5]:

$$
\int_{\partial \Omega} \frac{\partial G}{\partial x_{i}}\left(x, x_{0}\right) \frac{\partial^{2} G}{\partial y_{j} \partial v_{x}}\left(x, x_{0}\right)=-\frac{1}{2} \frac{\partial^{2} R}{\partial x_{i} \partial x_{j}}\left(x_{0}\right),
$$


and then obtain

$$
\lim _{k \rightarrow+\infty} \delta_{k}^{-1} \int_{\partial \Omega} \frac{\partial v_{k}}{\partial x_{i}} \frac{\partial w_{k}}{\partial v}=-8 \pi^{2} \sum_{j=1}^{2} a_{j} \frac{\partial^{2} R}{\partial x_{i} \partial x_{j}}\left(x_{0}\right) .
$$

We note here that (13) is shown by the Pohozaev identity [10].

Therefore, if we can show

$$
\lim _{k \rightarrow+\infty} \delta_{k}^{-1} \int_{\Omega} \frac{\partial \log V}{\partial x_{i}} \cdot \lambda_{k} V e^{v_{k}} \cdot w_{k}=2 \pi \sum_{j=1}^{2} a_{j} \frac{\partial^{2} \log V}{\partial x_{i} \partial x_{j}}\left(x_{0}\right),
$$

then

$$
\sum_{j=1}^{2} a_{j}\left\{\frac{\partial^{2} R}{\partial x_{i} \partial x_{j}}\left(x_{0}\right)+\frac{1}{4 \pi} \frac{\partial^{2} \log V}{\partial x_{i} \partial x_{j}}\left(x_{0}\right)\right\}=0
$$

follows for $i=1,2$, and hence $a_{1}=a_{2}=0$ from the assumption.

For this purpose, we use the Taylor expansion around $x_{k}=\left(x_{k 1}, x_{k 2}\right)$ for large $k$ and obtain

$$
\begin{gathered}
\frac{\partial \log V}{\partial x_{i}}(x)=\frac{\partial \log V}{\partial x_{i}}\left(x_{k}\right)+\left[\left(x_{1}-x_{k 1}\right) \frac{\partial}{\partial x_{1}}+\left(x_{2}-x_{k 2}\right) \frac{\partial}{\partial x_{2}}\right] \\
\cdot \frac{\partial \log V}{\partial x_{i}}\left(x_{k}\right)+R_{k}(x)\left|x-x_{k}\right|
\end{gathered}
$$

for $x=\left(x_{1}, x_{2}\right)$ with $\left|R_{k}(x)\right| \leq r\left(x, x_{k}\right)$, where $r\left(\cdot, x_{k}\right)$ is uniformly bounded on $\bar{\Omega}$, and near $x_{0}$,

$$
r\left(x, x_{k}\right)=\sup _{y \in B\left(x_{k},\left|x-x_{k}\right|\right)} \sum_{i, j}\left|\frac{\partial^{2} \log V}{\partial x_{i} \partial x_{j}}(y)-\frac{\partial^{2} \log V}{\partial x_{i} \partial x_{j}}\left(x_{k}\right)\right| .
$$

Therefore, this $r\left(\cdot, x_{k}\right)$ is continuous there, satisfying $r\left(x_{k}, x_{k}\right)=0$ and converging to $r\left(\cdot, x_{0}\right)$ uniformly. We shall show that there exists $C_{3}>0$ such that

$$
\delta_{k}^{-1}\left|\left(x-x_{k}\right) w_{k}(x)\right| \leq C_{3}
$$

for any $x \in \bar{\Omega}$ and $k=1,2, \ldots$ Then, we have

$$
\left|\int_{\Omega} R_{k}(x)\right| x-x_{k}\left|\lambda_{k} V e^{v_{k}} \delta_{k}^{-1} w_{k}\right| \leq C_{3} \int_{\Omega} r\left(x, x_{k}\right) \lambda_{k} V e^{v_{k}} \rightarrow 0
$$

by $\lambda_{k} V e^{v_{k}} d x \rightarrow 8 \pi \delta_{x_{0}}(d x)$ and $r\left(x_{0}, x_{0}\right)=0$, and therefore the contribution of the residual term of (15) is neglected in the limit of (12). 
To show (16), we use

$$
w_{k}(x)=\mathrm{I}_{1, k}(x)+\mathrm{I}_{2, k}(x)
$$

with

$$
\begin{aligned}
& \delta_{k}^{-1} \mathrm{I}_{1, k}(x)=-\sum_{j=1}^{2} a_{j} \int_{\tilde{\Omega}_{k}} \frac{\partial G}{\partial y_{j}}\left(x, x_{k}+\delta_{k} y^{\prime}\right) \cdot g_{k}\left(y^{\prime}\right) d y^{\prime}, \\
& \delta_{k}^{-1} \mathrm{I}_{2, k}(x)=-\frac{b}{2} \sum_{j=1}^{2} \int_{\tilde{\Omega}_{k}} \frac{\partial G}{\partial y_{j}}\left(x, x_{k}+\delta_{k} y^{\prime}\right) \cdot y_{j}^{\prime} e^{u\left(y^{\prime}\right)} d y^{\prime} .
\end{aligned}
$$

There is $C_{4}>0$ such that

$$
\left|\frac{\partial G}{\partial y_{j}}(x, y)\right| \leq C_{4}|x-y|^{-1}
$$

for any $(x, y) \in \bar{\Omega} \times \bar{\Omega}$, and therefore

$\delta_{k}^{-1}\left|w_{k}(x)\right| \leq C_{4}\left(a_{1}+a_{2}+\frac{b}{2}\right) \cdot \int_{\tilde{\Omega}_{k}}\left|x-\delta_{k} y^{\prime}-x_{k}\right|^{-1}\left(\left|g_{k}\left(y^{\prime}\right)\right|+\left|y_{j}^{\prime}\right| e^{u\left(y^{\prime}\right)}\right) d y^{\prime}$

holds true. It is obvious that

$$
\left|g_{k}(y)\right|+\left|y_{j}\right| e^{u(y)} \leq C_{5}\left(1+|y|^{2}\right)^{-\frac{3}{2}}
$$

with $C_{5}>0$ independent of $y \in \mathbb{R}^{2}$ and $k=1,2, \ldots$, and hence

$$
\delta_{k}^{-1}\left|w_{k}(x)\right| \leq C_{4} C_{5}\left(a_{1}+a_{2}+\frac{b}{2}\right) \int_{\tilde{\Omega}_{k}}\left|x-\delta_{k} y^{\prime}-x_{k}\right|^{-1}\left(1+\left|y^{\prime}\right|^{2}\right)^{-\frac{3}{2}} d y^{\prime} .
$$

This implies

$\delta_{k}^{-1}\left|\left(\delta_{k} x^{\prime}\right) w_{k}\left(x_{k}+\delta_{k} x^{\prime}\right)\right| \leq C_{4} C_{5}\left(a_{1}+a_{2}+\frac{b}{2}\right) \int_{\mathbb{R}^{2}} \frac{\left|x^{\prime}\right|}{\left|x^{\prime}-y^{\prime}\right|}\left(1+\left|y^{\prime}\right|^{2}\right)^{-\frac{3}{2}} d y^{\prime}$,

but we have

$\int_{\mathbb{R}^{2}} \frac{\left|x^{\prime}\right|}{\left|x^{\prime}-y^{\prime}\right|}\left(1+\left|y^{\prime}\right|^{2}\right)^{-\frac{3}{2}} d y^{\prime}=\int_{0}^{2 \pi} d \theta \int_{0}^{\infty}\left|x^{\prime}\right|\left(1+\left|x^{\prime}+r e^{\imath \theta}\right|^{2}\right)^{-\frac{3}{2}} d r \leq C_{6}$ with $C_{6}>0$ independent of $x^{\prime} \in \mathbb{R}^{2}$. Hence (16) follows for $x \in \bar{\Omega}$ and $k=1,2, \ldots$ Thus, we have proven that the limit of the right-hand side of (12) is reduced to

$$
\lim _{k \rightarrow+\infty} \delta_{k}^{-1} \int_{\Omega} \frac{\partial \log V}{\partial x_{i}} \cdot \lambda_{k} V e^{v_{k}} \cdot w_{k}=\lim _{k \rightarrow+\infty}\left\{\mathrm{II}_{0, k}+\mathrm{II}_{1, k}+\mathrm{II}_{2, k}\right\},
$$


where

$$
\begin{aligned}
\mathrm{II}_{0, k} & =\frac{\partial \log V}{\partial x_{i}}\left(x_{k}\right) \int_{\Omega} \lambda_{k} V e^{v_{k}} \cdot \delta_{k}^{-1} w_{k}, \\
\mathrm{II}_{1, k} & =\frac{\partial^{2} \log V}{\partial x_{1} \partial x_{i}}\left(x_{k}\right) \int_{\Omega}\left(x_{1}-x_{k 1}\right) \cdot \lambda_{k} V e^{v_{k}} \cdot \delta_{k}^{-1} w_{k}, \\
\mathrm{II}_{2, k} & =\frac{\partial^{2} \log V}{\partial x_{2} \partial x_{i}}\left(x_{k}\right) \int_{\Omega}\left(x_{2}-x_{k 2}\right) \lambda_{k} V e^{v_{k}} \cdot \delta_{k}^{-1} w_{k} .
\end{aligned}
$$

First, we have

$$
\begin{gathered}
\mathrm{II}_{0, k}=-\frac{\partial \log V}{\partial x_{i}}\left(x_{k}\right) \int_{\Omega} \delta_{k}^{-1} \Delta w_{k}=-\frac{\partial \log V}{\partial x_{i}}\left(x_{k}\right) \int_{\partial \Omega} \delta_{k}^{-1} \frac{\partial w_{k}}{\partial v} \\
\rightarrow-\frac{\partial \log V}{\partial x_{i}}\left(x_{0}\right) \cdot 2 \pi \sum_{j=1}^{2} a_{j} \int_{\partial \Omega} \frac{\partial^{2} G}{\partial v_{x} \partial y_{j}}\left(\cdot, x_{0}\right)
\end{gathered}
$$

and

$$
\int_{\partial \Omega} \frac{\partial^{2} G}{\partial v_{x} \partial y_{j}}\left(\cdot, x_{0}\right)=\int_{\partial B_{r}\left(x_{0}\right)} \frac{\partial^{2} G}{\partial v_{x} \partial y_{j}}\left(\cdot, x_{0}\right)=\int_{\partial B_{r}\left(x_{0}\right)} \frac{\partial^{2} G_{0}}{\partial v_{x} \partial y_{j}}\left(\cdot, x_{0}\right)+o(1)
$$

as $r \downarrow 0$, where $G_{0}(x, y)=\frac{1}{2 \pi} \log \frac{1}{|x-y|}$. Then it holds that

$$
\frac{\partial^{2} G_{0}}{\partial v_{x} \partial y_{j}}\left(x, x_{0}\right)=-\frac{1}{2 \pi} \frac{x_{j}-x_{0 j}}{\left|x-x_{0}\right|^{3}}
$$

for $x \in \partial B_{r}\left(x_{0}\right)$, and therefore

$$
\int_{\partial B_{r}\left(x_{0}\right)} \frac{\partial^{2} G_{0}}{\partial v_{x} \partial y_{j}}\left(\cdot, x_{0}\right)=0 .
$$

Thus, we have proven $\lim _{k \rightarrow+\infty} \mathrm{II}_{0, k}=0$.

Next, we have

$$
\begin{aligned}
\int_{\Omega}( & \left.x_{\ell}-x_{k \ell}\right) \cdot \lambda_{k} V e^{v_{k}} \cdot w_{k}=-\int_{\Omega}\left(x_{\ell}-x_{k \ell}\right) \Delta w_{k} \\
& =\int_{\Omega} \frac{\partial w_{k}}{\partial x_{\ell}}-\int_{\partial \Omega}\left(x_{\ell}-x_{k \ell}\right) \frac{\partial w_{k}}{\partial v}=\int_{\partial \Omega}\left\{v_{\ell} w_{k}-\left(x_{\ell}-x_{k \ell}\right) \frac{\partial w_{k}}{\partial v}\right\} \\
& =-\int_{\partial \Omega}\left(x_{\ell}-x_{k \ell}\right) \frac{\partial w_{k}}{\partial v}
\end{aligned}
$$


for $\ell=1,2$, and this implies

$$
\begin{aligned}
\mathrm{II}_{\ell, k}=- & \frac{\partial^{2} \log V}{\partial x_{\ell} \partial x_{i}}\left(x_{k}\right) \int_{\partial \Omega}\left(x_{\ell}-x_{k \ell}\right) \delta_{k}^{-1} \frac{\partial w_{k}}{\partial v} \\
& \rightarrow-\frac{\partial^{2} \log V}{\partial x_{\ell} \partial x_{i}}\left(x_{0}\right) \cdot 2 \pi \sum_{j=1}^{2} a_{j} \int_{\partial \Omega}\left(x_{\ell}-x_{0 \ell}\right) \frac{\partial^{2} G}{\partial v_{x} \partial y_{j}}\left(\cdot, x_{0}\right) .
\end{aligned}
$$

Here, we have

$$
\begin{aligned}
\int_{\partial \Omega}( & \left.x_{\ell}-x_{0 \ell}\right) \frac{\partial^{2} G}{\partial v_{x} \partial y_{j}}\left(x, x_{0}\right)=\int_{\partial \Omega} \frac{\partial}{\partial v_{x}}\left\{\left(x_{\ell}-x_{0 \ell}\right) \frac{\partial G}{\partial y_{j}}\left(x, x_{0}\right)\right\} \\
= & \int_{\partial B_{r}\left(x_{0}\right)} \frac{\partial}{\partial v_{x}}\left\{\left(x_{\ell}-x_{0 \ell}\right) \frac{\partial G}{\partial y_{j}}\left(x, x_{0}\right)\right\} \\
& \quad+\int_{\Omega \backslash B_{r}\left(x_{0}\right)} \Delta\left[\left(x_{\ell}-x_{0 \ell}\right) \frac{\partial G}{\partial y_{j}}\left(x, x_{0}\right)\right] \\
= & \int_{\partial B_{r}\left(x_{0}\right)} \frac{\partial}{\partial v_{x}}\left\{\left(x_{\ell}-x_{0 \ell}\right) \frac{\partial G}{\partial y_{j}}\left(x, x_{0}\right)\right\}+2 \int_{\Omega \backslash B_{r}\left(x_{0}\right)} \frac{\partial^{2} G}{\partial x_{\ell} \partial y_{j}}\left(x, x_{0}\right) \\
= & \int_{\partial B_{r}\left(x_{0}\right)} \frac{\partial}{\partial v_{x}}\left\{\left(x_{\ell}-x_{0 \ell}\right) \frac{\partial G}{\partial y_{j}}\left(x, x_{0}\right)\right\}-2 \int_{\partial B_{r}\left(x_{0}\right)} v_{\ell} \frac{\partial G}{\partial y_{j}}\left(x, x_{0}\right) \\
= & \int_{\partial B_{r}\left(x_{0}\right)} \frac{\partial}{\partial v_{x}}\left\{\left(x_{\ell}-x_{0 \ell}\right) \frac{\partial G_{0}}{\partial y_{j}}\left(x, x_{0}\right)\right\}-2 \int_{\partial B_{r}\left(x_{0}\right)} v_{\ell} \frac{\partial G_{0}}{\partial y_{j}}\left(x, x_{0}\right)+o(1)
\end{aligned}
$$

as $r \downarrow 0$, and the first term of the right-hand side is equal to 0 because

$$
\frac{\partial}{\partial v_{x}}\left\{\left(x_{\ell}-x_{0 \ell}\right) \frac{\partial G_{0}}{\partial y_{j}}\left(x, x_{0}\right)\right\}=\frac{x_{\ell}-x_{0 \ell}}{r}\left[\frac{\partial G_{0}}{\partial y_{j}}\left(x, x_{0}\right)+r \frac{\partial^{2} G_{0}}{\partial r \partial y_{j}}\left(x, x_{0}\right)\right]=0
$$

in terms of $r=\left|x-x_{0}\right|$. On the other hand, the second term is equal to

$$
-\frac{1}{\pi} \int_{\partial B_{r}\left(x_{0}\right)} \frac{\left(x_{\ell}-x_{0 \ell}\right)\left(x_{j}-x_{0 j}\right)}{r^{3}}=-\delta_{j \ell}= \begin{cases}-1 & (\ell=j), \\ 0 & (\ell \neq j),\end{cases}
$$

and therefore

$$
\lim _{k \rightarrow+\infty} \mathrm{II}_{\ell, k}=2 \pi a_{\ell} \frac{\partial^{2} \log V}{\partial x_{\ell} \partial x_{i}}\left(x_{0}\right)
$$

holds for $\ell=1,2$. We obtain (14), and the proof is complete.

Once $a_{1}=a_{2}=0$ is obtained, then the proof of $b=0$ is similar to [5]. For the sake of completeness, we confirm the following lemma and conclude the proof of Theorem 1.4. 
Lemma 3.2. Under the assumptions of the previous lemma, it holds that $b=0$.

Proof. By Lemma 3.1, we have

$$
\tilde{w}_{k}(x) \longrightarrow b \frac{\frac{8}{c}-|x|^{2}}{\frac{8}{c}+|x|^{2}} \quad \text { in } C_{\mathrm{loc}}^{2, \alpha}\left(\mathbb{R}^{2}\right) .
$$

We assume $b \neq 0$ and note the equalities

$$
-w_{k} \Delta v_{k}=\lambda_{k} V e^{v_{k}} w_{k} \quad \text { and } \quad-v_{k} \Delta w_{k}=\lambda_{k} V e^{v_{k}} v_{k} w_{k}
$$

in $\Omega$ and also

$$
\int_{\Omega}\left(w_{k} \Delta v_{k}-v_{k} \Delta w_{k}\right)=\int_{\partial \Omega}\left(w_{k} \frac{\partial v_{k}}{\partial v}-v_{k} \frac{\partial w_{k}}{\partial v}\right)=0 .
$$

Then we have

$$
\lambda_{k} \int_{\Omega} V e^{v_{k}} w_{k}=\lambda_{k} \int_{\Omega} V e^{v_{k}} v_{k} w_{k}
$$

We also have

$$
\begin{aligned}
\lambda_{k} \int_{\Omega} V e^{v_{k}} v_{k} w_{k}= & \int_{\tilde{\Omega}_{k}} \tilde{V}_{k} e^{\tilde{e}_{k}} \tilde{v}_{k} \tilde{w}_{k}+\left\|v_{k}\right\|_{\infty} \lambda_{k} \int_{\Omega} V e^{v_{k}} w_{k} \\
= & \int_{\mathbb{R}^{2}} \frac{c}{\left(1+\frac{c}{8}|x|^{2}\right)^{2}} \cdot \log \frac{1}{\left(1+\frac{c}{8}|x|^{2}\right)^{2}} \cdot b \frac{\frac{8}{c}-|x|^{2}}{\frac{8}{c}+|x|^{2}} d x \\
& \quad+\left\|v_{k}\right\|_{\infty} \lambda_{k} \int_{\Omega} V e^{v_{k}} w_{k}+o(1) \\
= & 8 \pi b+\left\|v_{k}\right\|_{\infty} \lambda_{k} \int_{\Omega} V e^{v_{k}} w_{k}+o(1)
\end{aligned}
$$

by (7), and therefore

$$
8 \pi b=\left(1-\left\|v_{k}\right\|_{\infty}\right) \lambda_{k} \int_{\Omega} V e^{v_{k}} w_{k}+o(1)
$$

by (17).

We shall show

$$
\frac{\partial w_{k}}{\partial x_{i}}=o\left(\delta_{k}\right) \quad \text { locally uniformly in } \bar{\Omega} \backslash\left\{x_{0}\right\}
$$

for $i=1,2$ and

$$
\left\|v_{k}\right\|_{\infty}=-2 \log \lambda_{k}+2 \log \frac{8}{c}-8 \pi R\left(x_{0}\right)+o(1) .
$$


In fact, if this is the case we obtain $\lambda_{k} \sim \delta_{k}^{2}$ by (8), and therefore

$$
\left\|v_{k}\right\|_{\infty} \lambda_{k} \int_{\Omega} V e^{v_{k}} w_{k}=-\left\|v_{k}\right\|_{\infty} \int_{\partial \Omega} \frac{\partial w_{k}}{\partial v}=o\left(\delta_{k} \log \lambda_{k}\right)=o(1) .
$$

Then, $b=0$ follows from (18).

Proof of (19). In fact, we have

$$
\frac{\partial w_{k}}{\partial x_{i}}=\lambda_{k} \int_{\Omega} \frac{\partial G}{\partial x_{i}}(x, y) \cdot V(y) e^{v_{k}(y)} w_{k}(y) d y=\int_{\tilde{\Omega}_{k}} \frac{\partial G}{\partial x_{i}}\left(x, x_{k}+\delta_{k} y^{\prime}\right) h_{k}\left(y^{\prime}\right) d y^{\prime}
$$

with

$$
h_{k}(y)=\tilde{V}_{k}(y) e^{\tilde{v}_{k}(y)} \tilde{w}_{k}(y)=O\left(\frac{1}{|y|^{4}}\right)
$$

uniformly in $k$ and

$$
h_{k}(y) \rightarrow h_{0}(y)=64 b c \frac{8-c|y|^{2}}{\left(8+c|y|^{2}\right)^{3}}
$$

locally uniformly in $y \in \mathbb{R}^{2}$. Therefore $\zeta_{k}(y) \rightarrow \zeta_{0}(y)$ locally uniformly in $y \in \mathbb{R}^{2}$ for $\zeta_{k}=\zeta_{k}(y)$ defined in Lemma 6 of [5]:

$$
\zeta_{k}\left(y_{1}, y_{2}\right)=\log \left[\frac{1}{y_{1}^{2}+y_{2}^{2}} \int_{-\infty}^{\sqrt{y_{1}^{2}+y_{2}^{2}}} t h_{k}\left(\frac{t y_{1}}{\sqrt{y_{1}^{2}+y_{2}^{2}}}, \frac{t y_{2}}{\sqrt{y_{1}^{2}+y_{2}^{2}}}\right) d t\right] .
$$

Here we have

$$
\left(y_{1} \frac{\partial \zeta_{k}}{\partial y_{1}}+y_{2} \frac{\partial \zeta_{k}}{\partial y_{2}}+2\right) e^{\zeta_{k}}=h_{k}
$$

and

$$
\zeta_{0}(y)=\log \frac{32 b c}{\left(8+c|y|^{2}\right)^{2}},
$$

and the dominated convergence theorem guarantees

$$
\begin{aligned}
\frac{\partial w_{k}}{\partial x_{i}}(x) & =\left.\int_{\tilde{\Omega}_{k}} \frac{\partial G}{\partial x_{i}}\left(x, x_{k}+\delta_{k} y^{\prime}\right) \cdot\left(y_{1} \frac{\partial \zeta_{k}}{\partial y_{1}}+y_{2} \frac{\partial \zeta_{k}}{\partial y_{2}}+2\right) e^{\zeta k}\right|_{y=y^{\prime}} d y^{\prime} \\
& =-\sum_{j=1}^{2} \delta_{k} \int_{\tilde{\Omega}_{k}} \frac{\partial^{2} G}{\partial x_{i} \partial y_{j}}\left(x, x_{k}+\delta_{k} y^{\prime}\right) \cdot y_{j}^{\prime} e^{\zeta_{k}\left(y^{\prime}\right)} d y^{\prime} \\
& =-\delta_{k}\left\{\sum_{j=1}^{2} \frac{\partial^{2} G}{\partial x_{i} \partial y_{j}}\left(x, x_{0}\right) \int_{\mathbb{R}^{2}} \frac{32 b c y_{j}^{\prime}}{\left(8+c\left|y^{\prime}\right|^{2}\right)^{2}} d y^{\prime}+o(1)\right\}=o\left(\delta_{k}\right)
\end{aligned}
$$

and hence (19). 
Proof of (20). We have $G(x, y)=\frac{1}{2 \pi} \log \frac{1}{|x-y|}+K(x, y)$ with $K \in C^{2, \alpha}(\Omega \times \bar{\Omega})$, and therefore it follows that

$$
\left\|v_{k}\right\|_{\infty}=v_{k}\left(x_{k}\right)=\mathrm{III}_{1, k}+\mathrm{III}_{2, k}
$$

where

$$
\begin{aligned}
\mathrm{III}_{1, k} & =-\frac{\lambda_{k}}{2 \pi} \int_{\Omega} \log \left|x_{k}-y\right| \cdot V(y) e^{v_{k}(y)} d y, \\
\mathrm{III}_{2, k} & =\lambda_{k} \int_{\Omega} K\left(x_{k}, y\right) V(y) e^{v_{k}(y)} d y .
\end{aligned}
$$

We have $\lambda_{k} V e^{v_{k}} d x \rightarrow 8 \pi \delta_{x_{0}}(d x)$, and therefore

$$
\mathrm{III}_{2, k}=8 \pi K\left(x_{0}, x_{0}\right)+o(1)=8 \pi R\left(x_{0}\right)+o(1) .
$$

For the first term, on the other hand, we have

$$
\begin{aligned}
\mathrm{III}_{1, k}= & -\frac{1}{2 \pi} \int_{\tilde{\Omega}_{k}} \log \left|\delta_{k} y^{\prime}\right| \cdot \tilde{V}_{k}\left(y^{\prime}\right) e^{\tilde{v}_{k}\left(y^{\prime}\right)} d y^{\prime} \\
= & \frac{1}{4 \pi}\left(\log \lambda_{k}+\left\|v_{k}\right\|_{\infty}\right) \int_{\tilde{\Omega}_{k}} \tilde{V}_{k}\left(y^{\prime}\right) e^{\tilde{v}_{k}\left(y^{\prime}\right)} d y^{\prime} \\
& \quad-\frac{1}{2 \pi} \int_{\tilde{\Omega}_{k}} \log \left|y^{\prime}\right| \cdot \tilde{V}_{k}\left(y^{\prime}\right) e^{\tilde{v}_{k}\left(y^{\prime}\right)} d y^{\prime}
\end{aligned}
$$

by (8), and therefore an asymptotic formula of [3] guarantees

$$
\int_{\tilde{\Omega}_{k}} \tilde{V}_{k}\left(y^{\prime}\right) e^{\tilde{v}_{k}\left(y^{\prime}\right)} d y^{\prime}=\int_{\Omega} V_{k}(y) e^{v_{k}(y)} d y=8 \pi+O\left(\lambda_{k}\left|\log \lambda_{k}\right|\right) .
$$

Thus we obtain

$$
\begin{aligned}
\mathrm{III}_{1, k}= & \left(2 \log \lambda_{k}+2\left\|v_{k}\right\|_{\infty}\right)\left(1+O\left(\lambda_{k}\left|\log \lambda_{k}\right|\right)\right) \\
& -\frac{1}{2 \pi} \int_{\mathbb{R}^{2}}\left(\log \left|y^{\prime}\right|\right) \cdot \frac{c}{\left(1+\frac{c}{8}\left|y^{\prime}\right|^{2}\right)^{2}} d y^{\prime}+o(1) \\
= & \left(2 \log \lambda_{k}+2\left\|v_{k}\right\|_{\infty}\right)\left(1+O\left(\lambda_{k}\left|\log \lambda_{k}\right|\right)\right)-2 \log \frac{8}{c}+o(1) .
\end{aligned}
$$

These results are summarized as

$$
\begin{aligned}
\left\|v_{k}\right\|_{\infty} & \left(1+O\left(\lambda_{k}\left|\log \lambda_{k}\right|\right)\right) \\
& =-2\left(\log \lambda_{k}\right) \cdot\left(1+O\left(\lambda_{k}\left|\log \lambda_{k}\right|\right)\right)+2 \log \frac{8}{c}-8 \pi R\left(x_{0}\right)+o(1),
\end{aligned}
$$

or (20). 


\section{References}

[1] Brezis, H. and F. Merle, Uniform estimates and blow-up behavior for solutions of $-\Delta u=$ $V(x) e^{u}$ in two dimensions. Comm. Partial Differential Equations 16 (1991), 1223-1253. Zbl 0746.35006 MR 1132783

[2] C.-C. Chen and C.-S. Lin, On the symmetry of blowup solutions to a mean field equation. Ann. Inst. H. Poincaré, Analyse Non lineaire 18 (2001), 271-296. Zbl 0995.35004 MR 1831657

[3] C.-C. Chen and C.-S. Lin, Sharp estimates for solutions to multi-bubbles in compact Riemann surfaces. Comm. Pure Appl. Math. 55 (2002), 728-771. Zbl 1040.53046 MR 1885666

[4] C.-C. Chen and C.-S. Lin, Classification of solutions of some nonlinear elliptic equations. Duke Math. J. 63 (1991), 615-622. Zbl 0768.35025 MR 1121147

[5] F. Gladiali and M. Grossi, Some results for the Gel'fand problem. Comm. Partial Differential Equations 29 (2004), 1335-1364. Zbl 02130271 MR 2103839

[6] Y. Y. Li, Harnack type inequality: the method of moving planes. Comm. Math. Phys. 200 (1999), 421-444. Zbl 0928.35057 MR 1673972

[7] L. Ma, and J. C. Wei, Convergence for a Liouville equation. Comment. Math. Helv. 76 (2001), 506-514. Zbl 0987.35056 MR 1854696

[8] K. Nagasaki and T. Suzuki, Asymptotic analysis for two-dimensional elliptic eigenvalue problems with exponentially dominated nonlinearities. Asymptotic Analysis 3 (1990), 173-188. Zbl 0726.35011 MR 1061665

[9] H. Ohtsuka and T. Suzuki, Blow-up analysis for Liouville type equation in self-dual gauge field theories. Commun. Contemp. Math. 7 (2005), 177-205. Zbl 02186811 MR 2140549

[10] S. Pohozaev, Eigenfunctions of the equation $\Delta u+\lambda f(u)=0$. Soviet. Math. Dokl. 6 (1965), 1408-1411. Zbl 0141.30202 MR 0192184

Received September 15, 2004; revised November 29, 2005

Tomohiko Sato, Takashi Suzuki, Division of Mathematical Science, Department of System Innovation, Graduate School of Engineering Science, Osaka University,

1-3 Machikaneyama, Toyonaka, Osaka 560-8531 Japan

E-mail:suzuki@sigmath.es.osaka-u.ac.jp 